\title{
Antitumor activity of fucoidan: a systematic review and meta-analysis
}

\author{
Ling-Min Cao" ${ }^{1 \#, ~ Z a o-X i ~ S u n ~}{ }^{2 \#}$, Emmanuel Costantine Makale ${ }^{2}$, Guan-Kui Du ${ }^{3}$, Wen-Fang Long ${ }^{4}$, \\ Hai-Rong Huang ${ }^{1}$
}

${ }^{1}$ Department of Epidemiology, School of Public Health, Hainan Medical University, Haikou, China; ${ }^{2}$ Department of Surgery, The First Affiliated Hospital, National Clinical Skills Experimental Teaching Demonstration Center, Hainan Medical University, Haikou, China; ${ }^{3}$ Department of Biochemistry and Molecular Biology, Hainan Medical University, Haikou, China; ${ }^{4}$ Laboratory of Tropical Environment and Health, School of Public Health, Hainan Medical University, Haikou, China

Contributions: (I) Conception and design: L Cao, H Huang, Z Sun; (II) Administrative support: H Huang; (III) Provision of study materials: L Cao; (IV) Collection and assembly of data: L Cao, H Huang, W Long, G Du; (V) Data analysis and interpretation: L Cao, H Huang, Z Sun, EC Makale, W Long, G Du; (VI) Manuscript writing: All authors; (VII) Final approval of manuscript: All authors.

\#These authors contributed equally to this work.

Correspondence to: Hai-Rong Huang. Department of Epidemiology, School of Public Health, Hainan Medical University, No. 3 Xueyuan Road, Longhua District, Haikou, 571199, China. Email: jialidog1@163.com.

Objective: To systematically review the antitumor activity of fucoidan based on the results of animal experimental studies.

Methods: The databases of the Cochrane Library, PubMed, Ovid MEDLINE, Web of Science, Embase, China National Knowledge Infrastructure (CNKI), Sino Med, Wanfang, and Chinese Science and Technology Periodicals (CQVIP) were searched for randomized and controlled animal experiments on the antitumor activity of fucoidan. The search included studies published up to 31 December 2020, and there was no limit to the start date. Endnote X9 software was employed to manage and screen the literature, Systematic Review Centre for Laboratory Animal Experimentation (SYRCLE) was used for assessment of risk of bias, and RevMan 5.3 software was used for meta-analysis.

Results: A total of 23 articles were included in the study. The results showed that compared with the control group, the fucoidan intervention group had significantly inhibited tumor weight, volume, and number. The combined effect values were mean difference (MD) $=-0.94,95 \%$ confidence interval (CI): -1.10 to $-0.79 ; \mathrm{MD}=-0.78,95 \% \mathrm{CI}:-1.06$ to -0.50 ; and standardized mean difference $(\mathrm{SMD})=-3.27,95 \%$ CI: -4.30 to -2.23 , respectively. The results of subgroup analysis showed that low-dose and intragastric administration of fucoidan had the best effect on breast cancer in controlling tumor weight, low-dose and intraperitoneal injection had the best effect on multiple myeloma in controlling tumor volume, and highdose and intraperitoneal injection of fucoidan had the best effect on melanoma in controlling the number of tumors.

Conclusions: The existing evidence shows that fucoidan inhibits the growth and spontaneous metastasis of tumors in numerous animal models. The tumor type, dosage, and administration method have been shown to influence the effect of fucoidan, and thus its mechanism warrants further research. As the design quality of the included studies was not high, heterogeneity and bias may have affected the accuracy of the results.

Keywords: Fucoidan; animal tumor model; inhibition of tumor; meta-analysis

Submitted Aug 24, 2021. Accepted for publication Nov 21, 2021.

doi: $10.21037 /$ tcr-21-1733

View this article at: https://dx.doi.org/10.21037/tcr-21-1733 


\section{Introduction}

Cancer is the one of the leading causes of death in developing countries, second only to cardiovascular and cerebrovascular diseases (1). Cancer prevention and treatment have become the focus of researchers all over the world. At present, chemotherapy and radiotherapy are the most common cancer treatments; however, their adverse side effects should not be underestimated $(2,3)$. Cumulative researches of novel cancer treatments have shown that natural polysaccharide plays an important antitumor role due to its remarkable results, low toxicity, and minimal side effects (4). As a result, natural compounds with antitumor effects isolated from marine resources have been attracting considerable attention from the healthcare and pharmaceutical industries (5).

Fucoidan, also known as sulfated fucans, fucoidin, and fucan sulfate, was first extracted from palmate kelp by Kylin in 1913 (6). Fucoidan is a macromolecular polysaccharide rich in sulfate groups widely found in brown algae, such as Fucus vesiculosus, kelp, and Sargassum (5). Due to its range of biological effects, including antitumor, antioxidative, antiviral, anti-inflammatory, anticoagulation, and immunoregulatory activities (7), fucoidan has been considered to have great clinical potential.

To date, many animal experiments (8-30) and in vitro experiments (31-33) have been conducted in exploration of the antitumor effect of fucoidan. Tumor types have included colorectal cancer $(8,12,16)$, breast cancer $(9,14,15,23,28)$, prostate cancer $(10,18)$, ovarian cancer (11), lung cancer $(13,19-21,24,27)$, liver cancer $(17,22)$, melanoma $(21,25)$, multiple myeloma (26), and sarcoma $(29,30)$. Among the animal experiments, we found that there were differences in the design, intervention protocols, and results. To the best of our knowledge, there has been only 1 clinical trial of the antitumor effect of fucoidan involving human participants. Hsiang et al. were the first to conduct a clinical trial of low molecular weight fucoidan as a first-line treatment for patients with metastatic colorectal cancer (34). In their study, the disease control rate was significantly improved by low-molecular weight fucoidan combined with chemotherapy plus target agents.

There are 3 main anticancer mechanisms of fucoidan. First, fucoidan can induce apoptosis, affect the normal mitosis of cancer cells, and inhibit cancer cell proliferation by regulating the growth cycle of cancer cells. Second, fucoidan can inhibit the formation of vascular endothelial growth factor and tumor angiogenesis, cut off the source of nutrient supply to the tumor, and starve the tumor so as to block the diffusion and metastasis of cancer cells. Third, fucoidan can activate the immune system and enhance the ability of natural killer (NK) cells and T cells to kill tumor cells (35).

By conducting a review of the existing evidence for the antitumor activities of fucoidan, this study aimed to reduce the risk of transposing animal experiment results to clinical practice, enhance the guiding value of animal experiments to clinical research, reduce repetitive and excessive animal experiments, and facilitate implementation of the principle of "reduction, substitution, and optimization". Based on the results of animal experiments, we systematically reviewed the antitumor activity of fucoidan so as to provide comprehensive guidance for clinical trials. To the best of our knowledge, no systematic review on the antitumor activity of fucoidan has been reported previously.

We present the following article in accordance with the PRISMA reporting checklist (available at https://dx.doi. org/10.21037/tcr-21-1733).

\section{Methods}

\section{Inclusion criteria}

(I) Type of study: randomized and controlled trials involving animals.

(II) Subjects: cancer model mice, without limitation to the specific disease model or modelling methods.

(III) Intervention and control measures: intervention measure was fucoidan alone, excluding fucoidan combined with other drugs, the control group was treated with either physiological saline or phosphatebuffered saline (PBS).

(IV) Outcome indicators: tumor weight, tumor volume, and tumor number.

(V) Literature language: language was restricted to articles published in either the Chinese or English language.

\section{Exclusion criteria}

Duplicate publication or research with the same data; reviews, conference abstracts, letters for articles, and editorials; and literature with incorrect data.

\section{Retrieval strategy}

The databases of the Cochrane Library, PubMed, Ovid MEDLINE, Web of Science, Embase, China National 
Knowledge Infrastructure (CNKI), Sino Med, Wanfang Database, and Chinese Science and Technology Periodicals (CQVIP) were searched for studies on the antitumor effects of fucoidan. The search time was from the establishment of each database to 31 December 2020. In addition, applicable references from selected studies were also included. The search included combinations of medical subject heading $(\mathrm{MeSH})$ terms and key words. Search terms included Fucoidan, sulfated fucans, Fucoidin, Fucan sulfate, Mekabu fucoidan, fucan sulfate Hor-1, Neoplasms, Tumor, Cancer, Malignancy, Mice, Mus, Mouse, Rats, Rat, Rattus, Animal Experimentation, Animal Research, and Animal Experiment.

Specific retrieval strategy (taken from the PubMed database as an example):

(I) "fucoidan" [Supplementary Concept];

(II) sulfated fucans OR fucan sulfate OR fucoidin OR Mekabu fucoidan OR meFucoidan OR fucan sulfate Hor-1;

(III) \#1 OR \#2;

(IV) "Neoplasms"[Mesh];

(V) Neoplasia OR Neoplasias OR Neoplasm OR Tumors OR Tumor OR Cancer OR Cancers OR Malignancy OR Malignancies OR Malignant Neoplasms OR Malignant Neoplasm OR Benign Neoplasms OR Benign Neoplasm;

(VI) \#4 OR \#5;

(VII) "Mice"[Mesh];

(VIII) Mus OR Mouse OR Rats OR Rat OR Rattus OR Animal Experimentation OR Animal Research OR Animal Experiment;

(IX) \#7 OR \#8;

(X) \#3 AND \#6 AND \#9 (Filters: from 1981-2020).

\section{Literature screening and data extraction}

The software Endnote X9 (Clarivate Analytics, London, UK) was used by 2 trained reviewers to screen the literature strictly according to the inclusion and exclusion criteria. First, on the basis of reading the titles and abstracts of the literature, any article that obviously did not meet the inclusion criteria was eliminated. The full text of the remaining literature was then screened again according to the inclusion and exclusion criteria. If any differences arose between the 2 reviewers, a resolution was sought through discussion and analysis. If a consensus was not reached, a third reviewer was consulted before a decision was made regarding inclusion.
The data extracted from the included studies included the following: (I) title, first author, publication year, and author nationality; (II) animal species, weight, age, sample size, and cancer type; (III) fucoidan source, extraction method, intervention components, purity and dosage, administration method, intervention time, and intervention measures of control group; and (IV) tumor weight, tumor volume, and tumor number.

\section{Bias risk assessment}

The Systematic Review Centre for Laboratory Animal Experimentation (SYRCLE) risk of bias tool (36) was used to evaluate the included literature. This tool lists 10 items concerning different forms of bias, including selectivity bias, implementation bias, measurement bias, loss of follow-up bias, reporting bias, and others (36).

\section{Statistical analysis}

In accordance with the Cochrane Handbook for Systematic Reviews of Interventions (37), the data (mean \pm standard deviation) of multiple intervention groups were merged, and then the results were statistically analyzed using RevMan 5.3 software (Copenhagen: The Nordic Cochrane Center, The Cochrane Collaboration, 2014). Heterogeneity analysis was carried out on the included literatures. If there was no obvious heterogeneity among the included studies $\left(\mathrm{I}^{2} \leq 50 \%\right.$, $\mathrm{P} \geq 0.10$ ), a fixed effect model (FEM) was used for the meta-analysis. If there was heterogeneity between studies $\left(\mathrm{I}^{2}>50 \%, \mathrm{P}<0.10\right)$, the random effect model $(\mathrm{REM})$ was used for meta-analysis. Subgroup and sensitivity analyses were used to explore the potential sources of heterogeneity and evaluate the reliability of the results. Mean difference (MD) and 95\% confidence interval (CI) were calculated for the continuous data. Standardized mean difference (SMD) and $95 \%$ CI were used to calculate the results with different measurement methods, different measurement units or large differences. When at least 10 studies were included, a funnel chart was used to evaluate publication bias.

Subgroup analysis was proposed for the following: (I) different dosages of fucoidan (low, medium, and high); (II) different administration methods of fucoidan (intraperitoneal injection, tail vein injection, intragastric administration, and oral administration); (III) different cancer types (lung cancer, liver cancer, breast cancer, melanoma, sarcoma, colorectal cancer, multiple myeloma, prostate cancer, and ovarian cancer); and (IV) different 
mouse types (nude mice and non-nude mice).

\section{Results}

\section{Literature retrieval results}

A total of 1,187 papers were initially retrieved. Using Endnote X9 software and manual duplicate checking, 600 duplicate documents were eliminated. According to the inclusion and exclusion criteria, a further 564 studies were excluded after reading the title, abstract, and full text of the remaining literature. Finally, 11 English (8-18) and 12 Chinese (19-30) language studies were included. Among the 23 papers, there were 2 dissertations $(21,30)$, and each dissertation involved 2 independent studies. After completion of the screening process, a total of 23 articles involving 25 studies were included in the meta-analysis. The literature screening process is shown in Figure 1.

\section{Basic characteristics of the included studies}

The basic characteristics of the included studies are shown in Table 1. Among them, 3 studies used BALB/c mice $(9,15,28), 7$ studies used nude mice $(8,10,11,13,16-18), 3$ studies used Sprague-Dawley (SD) rats $(12,14,23), 7$ studies used C57BL/6J mice (19-21,24,25,27), 1 used Institute of Cancer Research (ICR) mice (22), 1 used nonobese diabetic/severe combined immunodeficiency (NOD/SCID) mice (26), and 3 used Kunming mice $(29,30)$. A total of 9 studies used males $(8,10-12,16,18,22,27,29), 12$ used females $(9,14,15,19,20,23-26,28,30)$, and 4 studies did not specify the gender of the mice $(13,17,21)$. Regarding the cancer models in each of the 25 studies, 3 were colorectal cancer $(8,12,16), 5$ were breast cancer $(9,14,15,23,28), 2$ were prostate cancer $(10,18), 1$ was ovarian cancer $(11)$, 6 were lung cancer $(13,19-21,24,27), 2$ were liver cancer $(17,22), 2$ were melanoma $(21,25), 1$ was multiple myeloma (26), and 3 were sarcoma $(29,30)$. Fucoidan sources included seaweed, vesicular brown algae, brown seaweed Sargassum fusiforme, American sea cucumber, sea melon, Icelandic sea cucumber, Arctic sea cucumber, glycoside moss, undaria spore leaf, and kelp. In 1 study, the intervention group was further specified as fucoidan FP08S2 (13), 3 were sea cucumber fucoidan sulfate (SC-FUC) $(19,24,25), 1$ was fucoidan from Sargassum SP (29), and 1 was lowmolecular weight fucoidan (LMWF) (30). There were 6 studies with only 1 intervention group $(8,10-12,17,20)$, 14 with 2 intervention groups $(9,13-16,18,19,21,23$ -
$26,28)$, and 5 with 3 intervention groups $(22,27,29,30)$. A total of 16 studies $(8,9,15,16,18-21,24-30)$ administered intraperitoneal fucoidan injection, 6 studies $(10-12,14,22,23)$ used intragastric administration, 2 studies $(17,30)$ involved oral administration, and 1 study (13) used caudal vein injection. There were 23 studies (8-17,19-25,27-30) with physiological saline in the control group and 2 studies $(18,26)$ with phosphate buffered saline (PBS). The outcome indexes of each study varied. Tumor weight was an outcome index in 22 studies $(9-15,17-27,29,30)$, tumor volume in 11 studies $(8-11,13,15-18,26,28)$, and tumor number in 7 studies $(12,19-21,24,25)$.

\section{Results of bias risk assessment}

The results of bias risk assessment are shown in Table 2 . All 25 controlled trials were randomly grouped, but it was unclear which specific randomization methods were used and whether appropriate methods were adopted to achieve the unpredictability of random sequences. None of the 23 articles mentioned whether covert grouping was implemented, and the information provided was insufficient to determine whether breeders and researchers were blinded. The animals were randomly placed in 11 studies $(8-12,14,15,17,18,26,30)$ and evaluated as "-". There was no experimental report on random selection of animals for result evaluation, and so the risk of bias was assessed as "+". None of the included studies blinded the evaluators of the results, however, this did not affect the determination of the indicators of the results (the outcome was an objective index), thus was evaluated as "_". The data of 17 studies (8-11,13,17-22,24-29) were reported completely.

\section{Meta-analysis}

A total of 22 studies $(9-15,17-27,29,30)$ reported tumor weight. Meta-analysis showed that $\mathrm{I}^{2}=98 \%$ among the studies, and a REM was used for combined analysis. Compared with the control group, fucoidan inhibited tumor weight (MD $=-0.94,95 \% \mathrm{CI}:-1.10$ to $-0.79, \mathrm{P}<0.05)$, and the difference was statistically significant.

A total of 11 studies $(8-11,13,15-18,26,28)$ reported tumor volume. Meta-analysis showed that $\mathrm{I}^{2}=99 \%$ among these studies, and REM was used for combined analysis. Compared with the control group, fucoidan inhibited tumor volume $(\mathrm{MD}=-0.78,95 \% \mathrm{CI}:-1.06$ to $-0.50, \mathrm{P}<0.05)$, and the difference was statistically significant.

A total of 7 studies $(12,19-21,24,25)$ reported the number 
Identification of studies via databases and registers
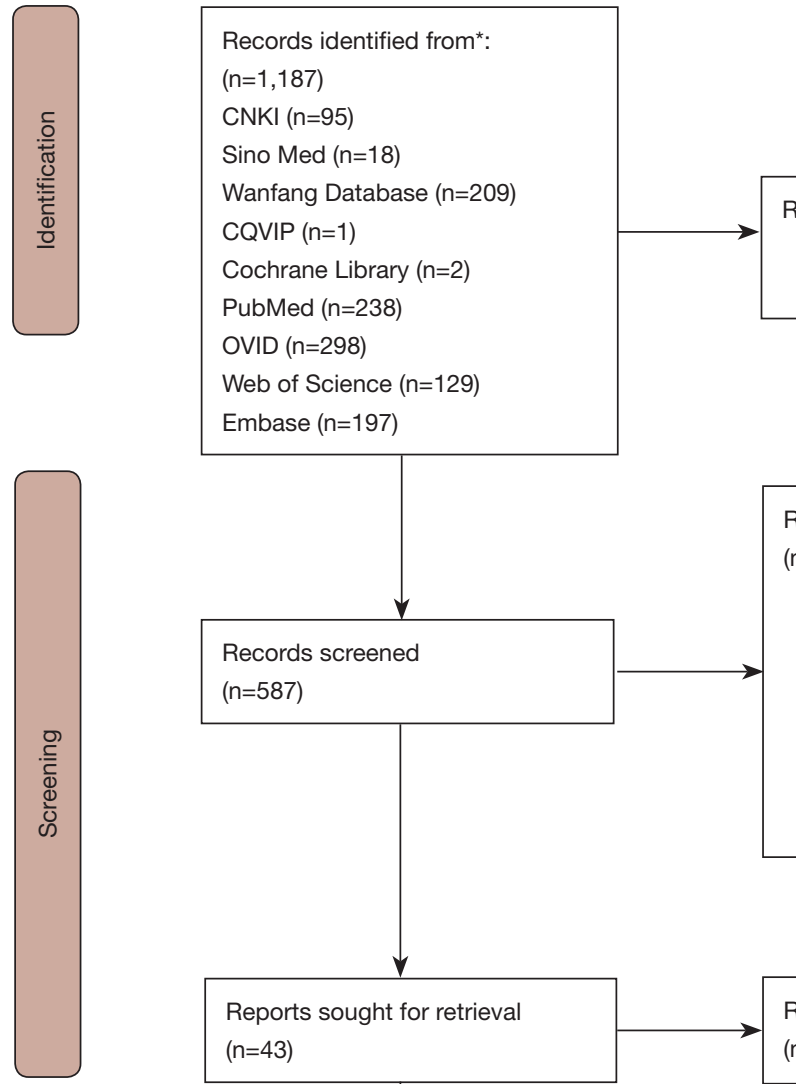

Records excluded ${ }^{\star *}$

$(\mathrm{n}=544)$

Intervention non-fucoidan $(n=132)$

Non-animal experimental study $(n=28)$

Irrelevant research $(n=274)$

Study on functions other than anti-tumor $(\mathrm{n}=87)$

The intervention measures in the control group were

not normal saline or phosphate buffer $(n=10)$

Review ( $n=13)$
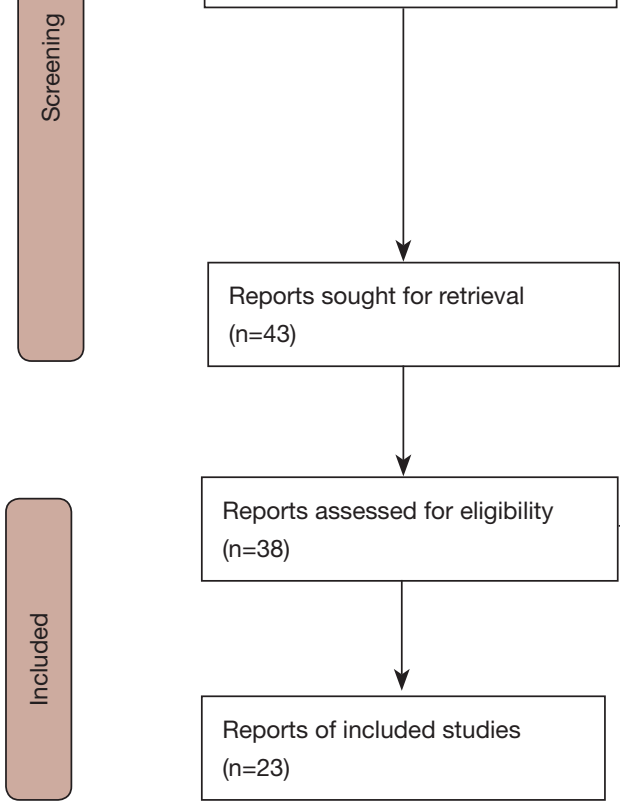

Reports not retrieved

$(n=5)$

Reports excluded:

$(n=15)$

Repeated publication of the same study $(n=3)$

Non-animal experimental study $(n=1)$

Intervention non-fucoidan $(\mathrm{n}=1)$

No quantitative outcome index $(n=3)$

No required outcome index $(n=4)$

Meeting report $(n=3)$

${ }^{*}$ Consider, if feasible to do so, reporting the number of records identified from each database or register searched (rather than the total number across all databases/registers).

**If automation tools were used, indicate how many records were excluded by a human and how many were excluded by automation tools.

From: Page MJ, McKenzie JE, Bossuyt PM, Boutron I, Hoffmann TC, Mulrow CD, et al. The PRISMA 2020 statement: an updated guideline for reporting systematic reviews. BMJ 2021;372:n71. doi: 10.1136/bmj.n71

Figure 1 Literature screening flow chart. 


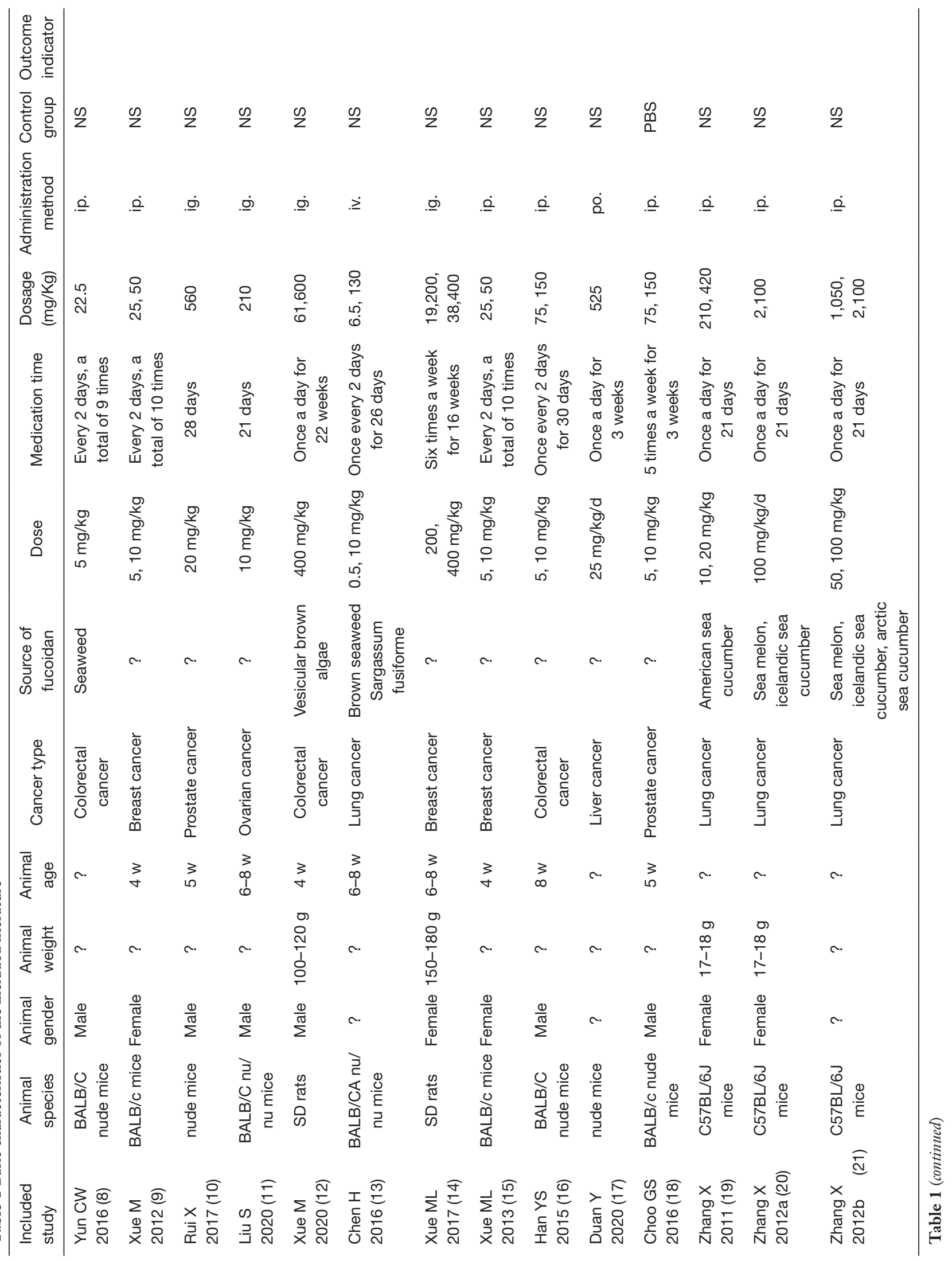




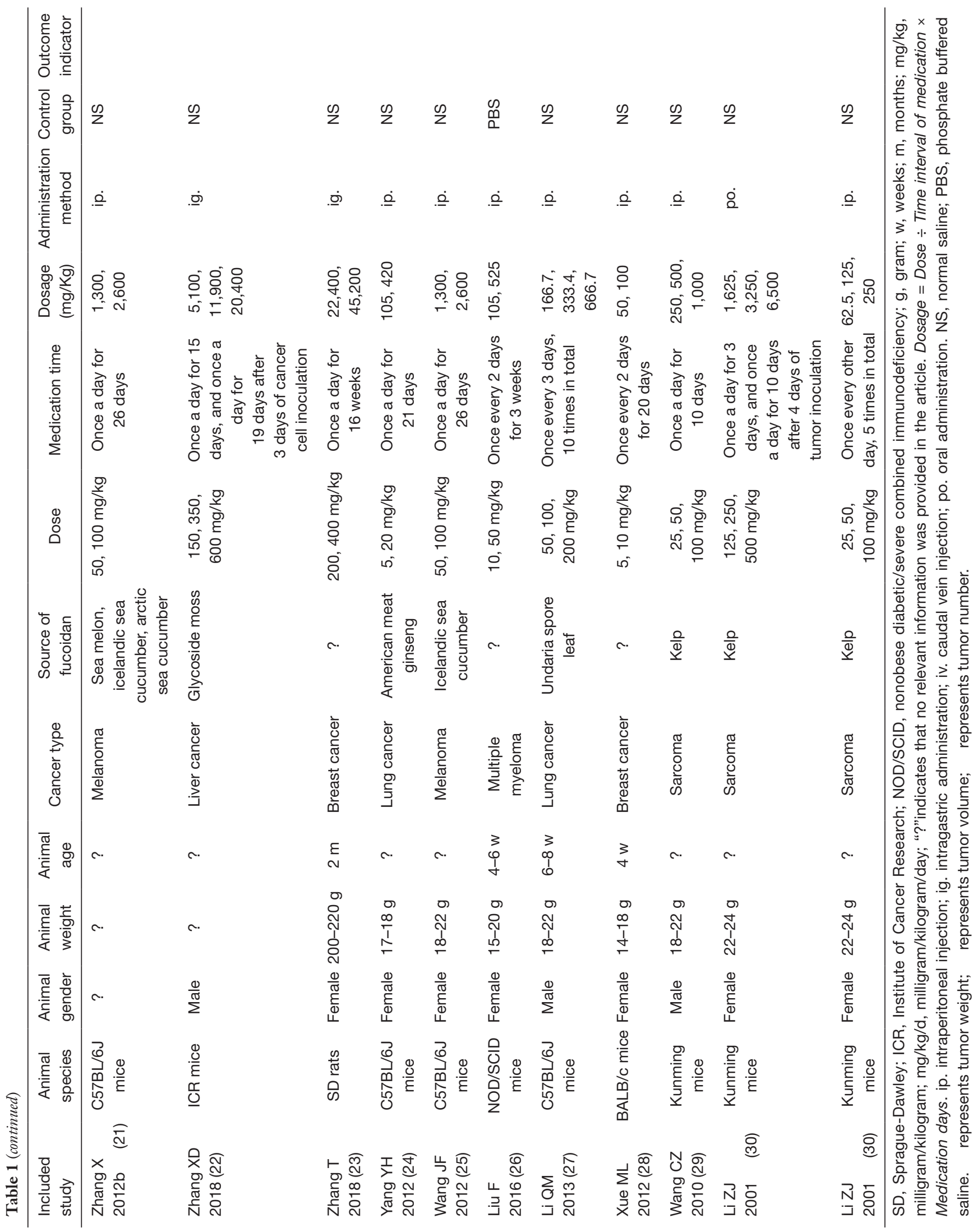


Table 2 SYRCLE bias risk assessment results of included studies

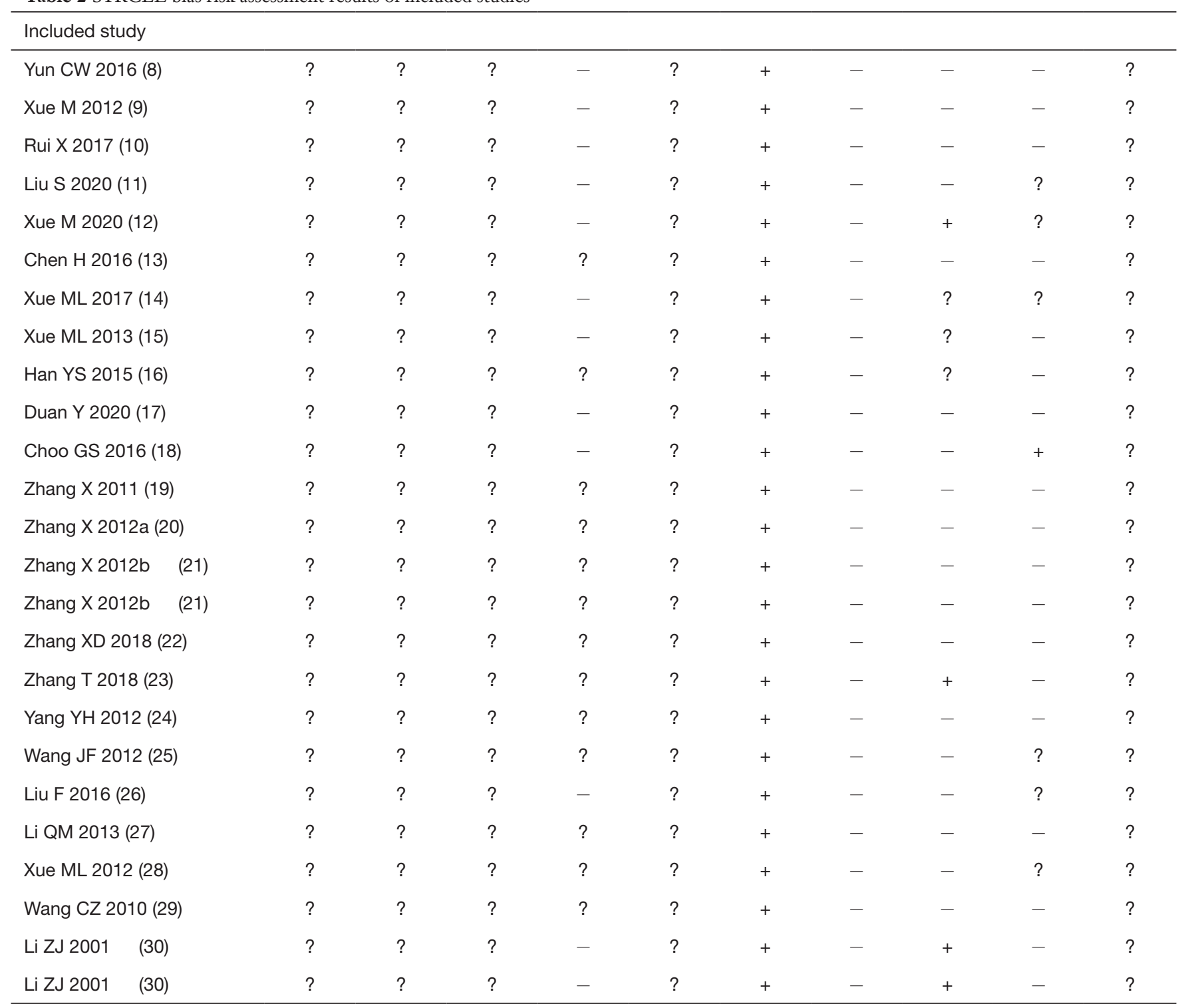

Evaluation of bias risk was based on the following determinations: (1) Whether the generation or application of allocation sequence was sufficient/correct; (2) Whether the baseline of each group was the same or whether confounding factors had been adjusted; (3) Whether allocation concealment was sufficient/correct; (4) Whether the animals were randomly placed during the experiment; (5) Whether animal breeders and researchers were blinded in the experiment to avoid knowing the intervention measures accepted by animals; (6) Whether the animals were randomly selected for outcome assessment; (7) Whether evaluators of results were blinded; (8) Whether incomplete data were fully/correctly explained; (9) Whether the research report had nothing to do with the selective result report; (10) Whether there were other issues that would lead to high risk of bias; "-" means low risk; "?” indicates uncertainty; "+" means high risk. 


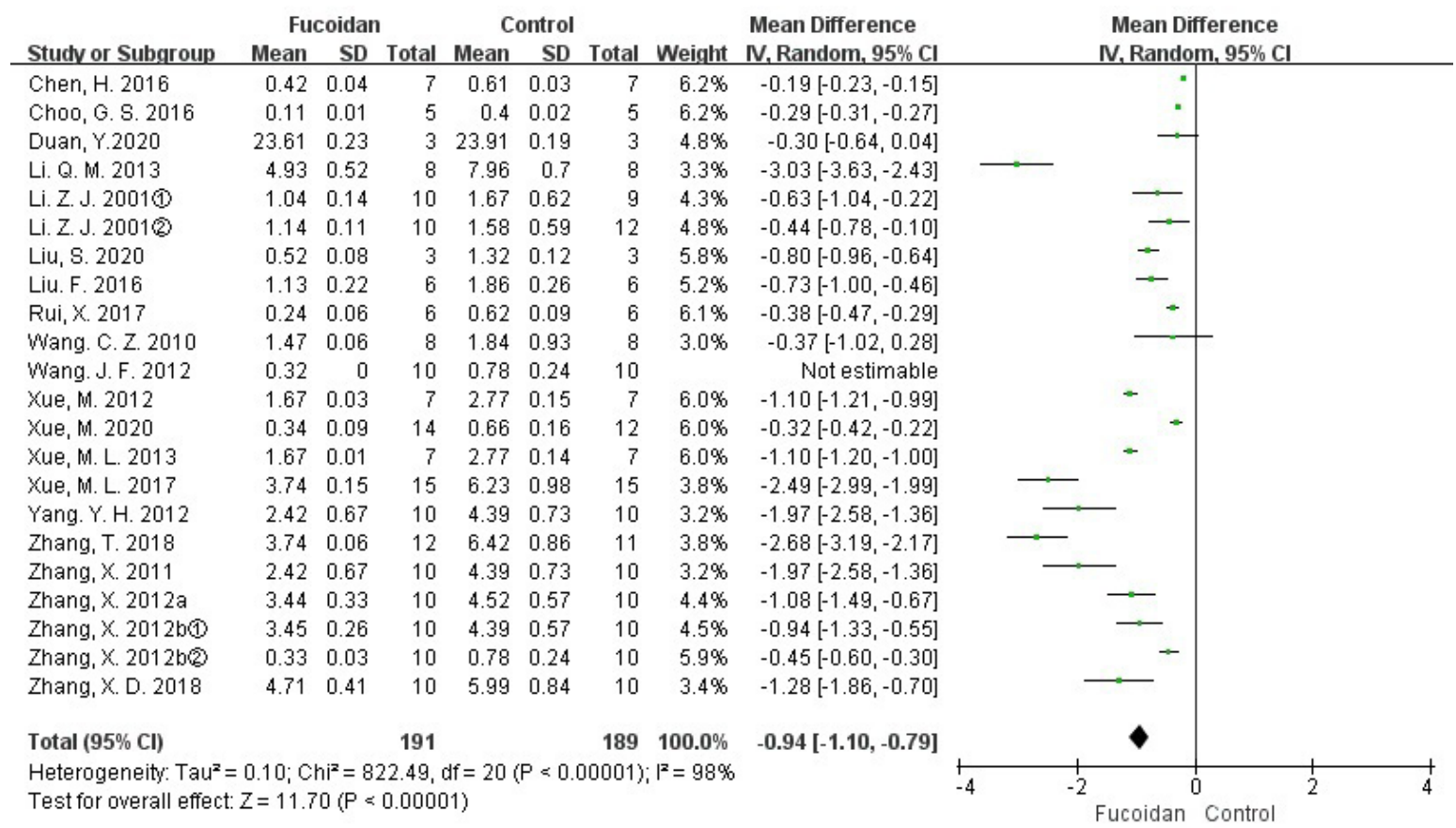

Figure 2 Meta-analysis results of tumor weight inhibition. SD, standard deviation; CI, confidence interval; IV, Inverse Variance; df, degree of freedom.

of tumors. As there were considerable differences in the outcome indicators of the various studies, the meta-analysis effect value was expressed by SMD and 95\% CI. Metaanalysis showed that $\mathrm{I}^{2}=73 \%$ among the studies, and REM was used for combined analysis. Compared with the control group, fucoidan inhibited tumor number $(\mathrm{SMD}=-3.27$, $95 \%$ CI: -4.30 to $-2.23, \mathrm{P}<0.05)$, and the difference was statistically significant.

The above results are shown in Figures 2-4, and Table 3.

\section{Analysis of source beterogeneity}

\section{Sensitivity analysis}

Based on tumor weight, tumor volume, and tumor number, the outcome indicators of the included literature were combined and analyzed by FEM and REM, and the analysis results of the 2 models were consistent. A total of 22 studies $(9-15,17-27,29,30)$ analyzed the tumor weight. After removing the studies 1 at a time, the $\mathrm{I}^{2}$ of each combination scheme was still between $97 \%$ and $98 \%$, and the heterogeneity had no obvious change. There were 11 studies $(8-11,13,15-18,26,28)$ which analyzed tumor volume. After deleting each study 1 at a time, the heterogeneity remained unchanged, and $\mathrm{I}^{2}$ was still $99 \%$. A total of 7 studies $(12,19-21,24,25)$ analyzed the number of tumors, and after removing the study by Wang Jingfeng (25), $\mathrm{I}^{2}$ decreased from $73 \%$ to $26 \%$, indicating it was a source of heterogeneity (Figure 5).

\section{Subgroup analysis}

In accordance with the Cochrane Handbook for Systematic Reviews of Interventions (7), because multiple intervention groups shared 1 control group, only the total number of subjects was divided in subgroup analysis, the original mean and standard deviation remaining unchanged. The results of the subgroup analysis are shown in Table 4.

Subgroup analysis of the effect of fucoidan intervention on tumor weight showed that the heterogeneity of studies in each dosage group was less than $50 \%$, and dosage was one of the variables affecting the heterogeneity among studies. The tumor weight in the intervention group was lower than that in the control group, with different dosages, administration modes, and mouse types, and the difference was statistically significant. Except for prostate cancer and ovarian cancer, the tumor weights of the other cancer types in the intervention group were lower than those in the control group, and the difference was statistically significant. 




Figure 3 Meta-analysis results of tumor volume inhibition. SD, standard deviation; CI, confidence interval; IV, Inverse Variance; df, degree of freedom.

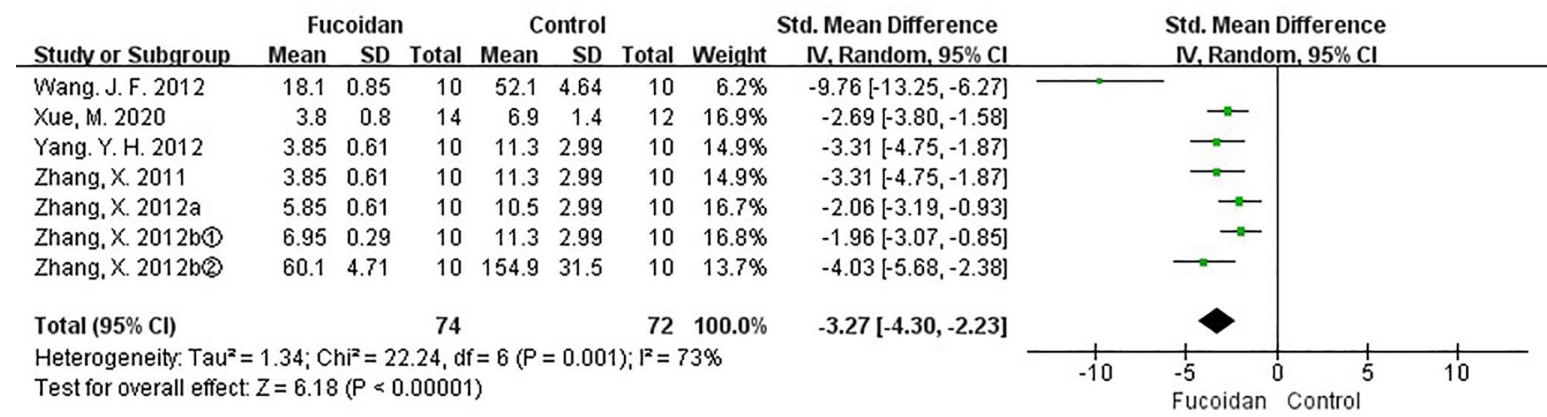

Figure 4 Meta-analysis results of tumor number inhibition. SD, standard deviation; CI, confidence interval; IV, Inverse Variance; df, degree of freedom; std, standard.

Table 3 Results of the meta-analysis of the tumor inhibition outcome index

\begin{tabular}{|c|c|c|c|c|c|c|}
\hline Outcome indicator & Number of studies & Sample size T/C & MD/SMD & $95 \% \mathrm{Cl}$ & $\mathrm{I}^{2} / \%$ & $P$ value \\
\hline Tumor volume $\left(\mathrm{cm}^{3}\right)$ & 11 & $67 / 67$ & $\mathrm{MD}=-0.78$ & $-1.06,-0.50$ & 99 & $<0.05$ \\
\hline Tumor number (pcs) & 7 & $74 / 72$ & SMD $=-3.27$ & $-4.30,-2.23$ & 73 & $<0.05$ \\
\hline
\end{tabular}

g, gram; $\mathrm{cm}^{3}$, cubic centimeter; pcs, pieces. T represents the intervention group, $\mathrm{C}$ represents the control group. MD, mean difference; $\mathrm{SMD}$, standardized mean difference; $\mathrm{Cl}$, confidence interval.

Subgroup analysis of the effect of fucoidan intervention on tumor volume showed that the heterogeneity of dosage, administration mode, and cancer type was less than 50\%, and these 3 variables affected the heterogeneity among studies. The tumor volume in the intervention group was smaller than that in the control group, with different dosages and mouse types, and the difference was statistically significant. Except for oral administration, there was no significant difference in tumor volume between treatment groups. The tumor volume of the administration methods other than oral in the intervention group was significantly lower than that in the control group. Except for lung 


\begin{tabular}{|c|c|c|c|c|c|c|c|c|c|c|c|c|}
\hline \multirow[b]{2}{*}{ Study or Subgroup } & \multicolumn{3}{|c|}{ Fucoidan } & \multicolumn{3}{|c|}{ Control } & \multicolumn{2}{|r|}{ Std. Mean Difference } & \multirow{2}{*}{\multicolumn{4}{|c|}{$\begin{array}{c}\text { Std. Mean Difference } \\
\text { IV, Random, } 95 \% \mathrm{Cl}\end{array}$}} \\
\hline & Mean & SD & Total & Mean & SD & Total & Weight & IV, Random, 95\% Cl & & & & \\
\hline Wang. J. F. 2012 & 18.1 & 0.85 & 10 & 52.1 & 4.64 & 10 & $0.0 \%$ & $-9.76[-13.25,-6.27]$ & & & & \\
\hline Xue, M. 2020 & 3.8 & 0.8 & 14 & 6.9 & 1.4 & 12 & $20.5 \%$ & $-2.69[-3.80,-1.58]$ & & $\rightarrow-$ & & \\
\hline Yang. Y. H. 2012 & 3.85 & 0.61 & 10 & 11.3 & 2.99 & 10 & $13.9 \%$ & $-3.31[-4.75,-1.87]$ & & & & \\
\hline Zhang, X. 2011 & 3.85 & 0.61 & 10 & 11.3 & 2.99 & 10 & $13.9 \%$ & $-3.31[-4.75,-1.87]$ & & - & & \\
\hline Zhang, $X .2012 a$ & 5.85 & 0.61 & 10 & 10.5 & 2.99 & 10 & $19.9 \%$ & $-2.06[-3.19,-0.93]$ & & $\rightarrow-$ & & \\
\hline Zhang, $X .2012 b(1)$ & 6.95 & 0.29 & 10 & 11.3 & 2.99 & 10 & $20.5 \%$ & $-1.96[-3.07,-0.85]$ & & $\rightarrow-$ & & \\
\hline Zhang, $X .2012 \mathrm{~b}(2)$ & 60.1 & 4.71 & 10 & 154.9 & 31.5 & 10 & $11.3 \%$ & $-4.03[-5.68,-2.38]$ & & & & \\
\hline Total $(95 \% \mathrm{Cl})$ & & & 64 & & & 62 & $100.0 \%$ & $-2.74[-3.35,-2.13]$ & & & & \\
\hline \multicolumn{9}{|c|}{$\begin{array}{l}\text { Heterogeneity: } \text { Tau }^{2}=0.15 ; \mathrm{Chi}^{2}=6.80, \mathrm{df}=5(P=0.24) ;\left.\right|^{2}=26 \% \\
\text { Test for overall effect: } Z=8.81(P<0.00001)\end{array}$} & -10 & $\begin{array}{l}-5 \\
\text { Fucoidan }\end{array}$ & Control & 10 \\
\hline
\end{tabular}

Figure 5 Sensitivity analysis results of tumor number inhibition. SD, standard deviation; CI, confidence interval; IV, Inverse Variance; df, degree of freedom; std, standard.

Table 4 Results of the meta-analysis of the tumor inhibition outcome index

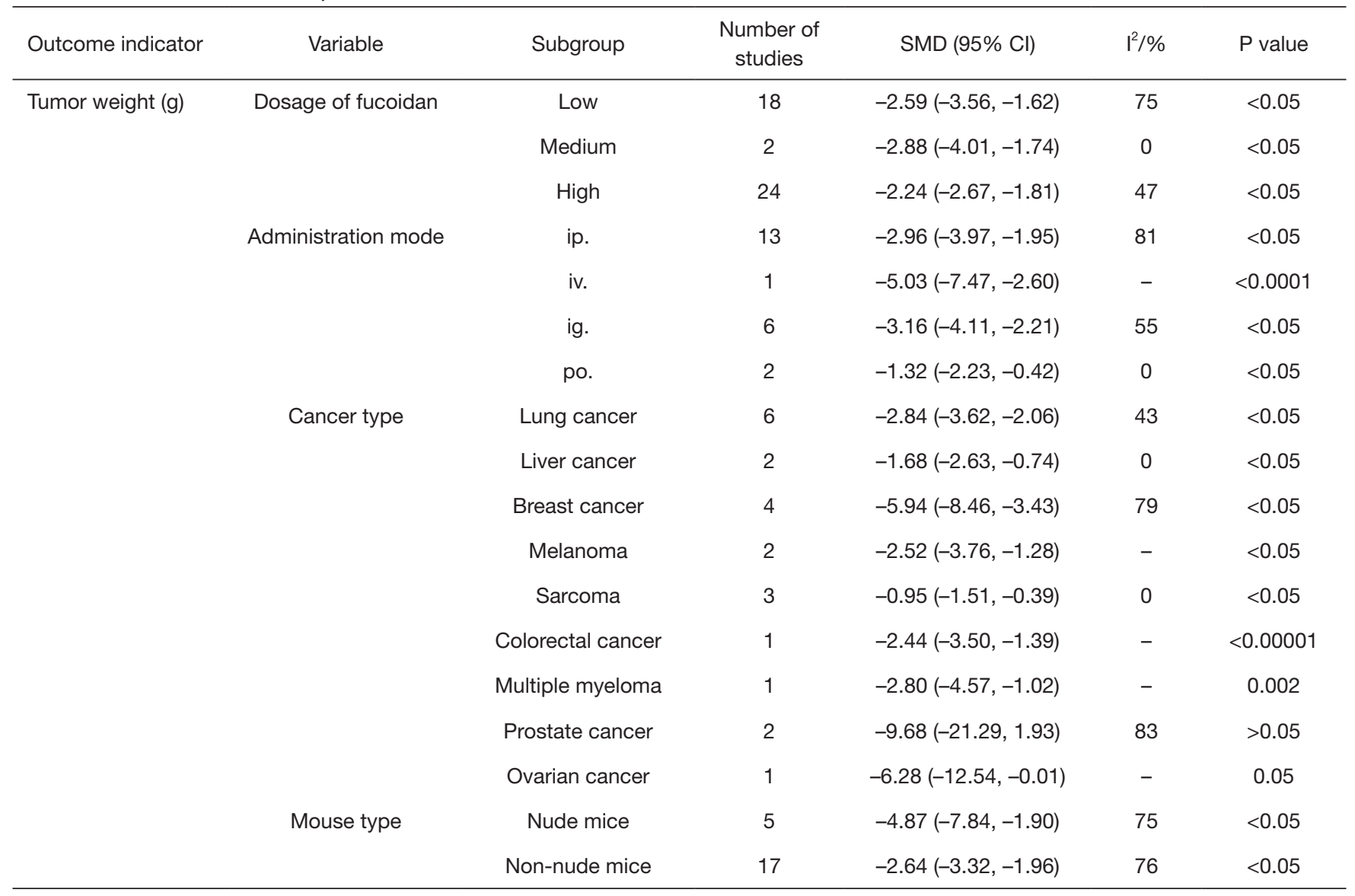

Table 4 (continued) 
Table 4 (continued)

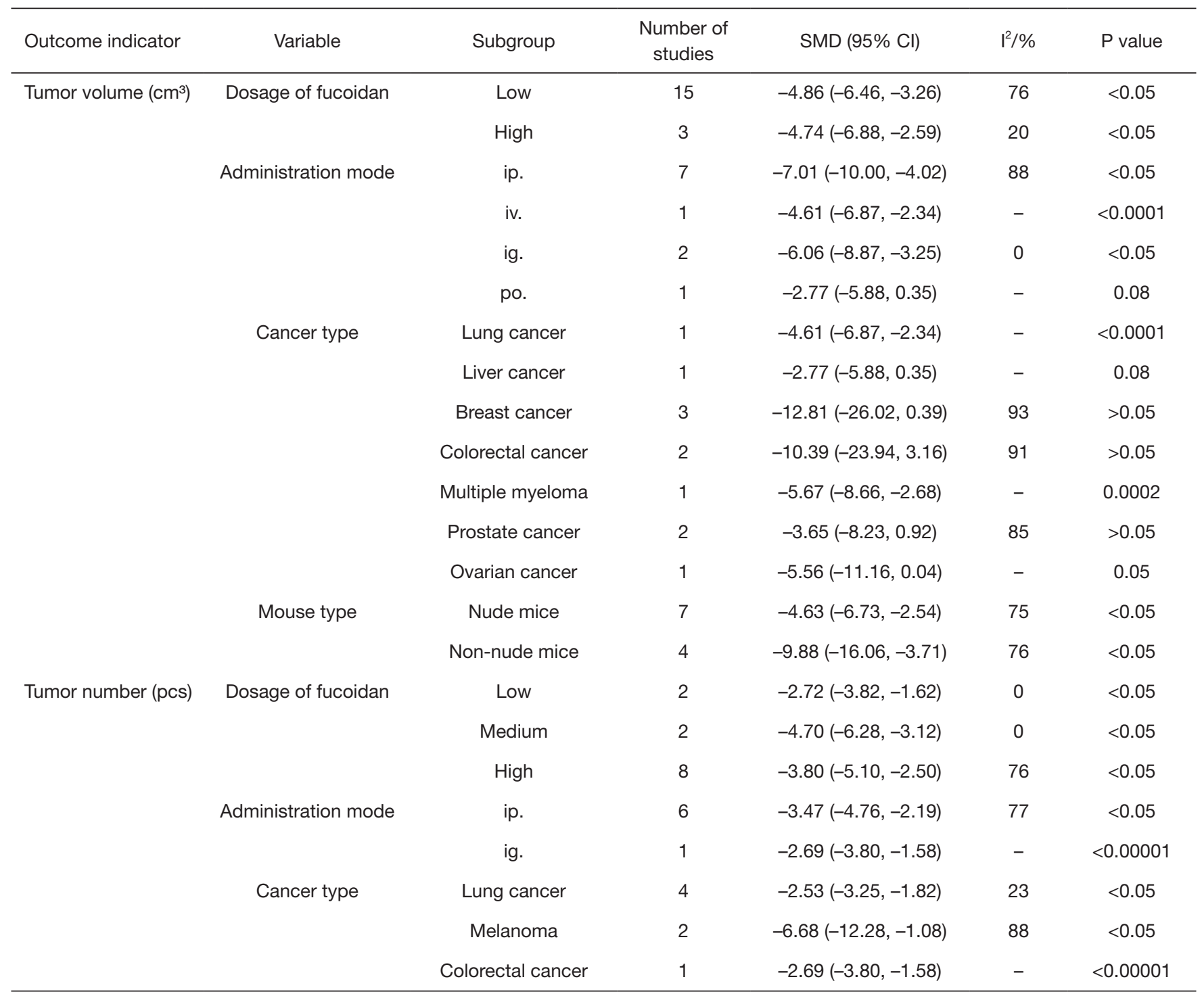

Dosages less than 420 mg/kg were considered low, 420 mg/kg medium, and greater than $420 \mathrm{mg} / \mathrm{kg}$ high. g, gram; cm³, cubic centimeter; pcs, pieces. ip. intraperitoneal injection; ig. intragastric administration; iv. caudal vein injection; po. oral administration. SMD, standardized mean difference; $\mathrm{Cl}$, confidence interval.

cancer and multiple myeloma, the tumor volume in the intervention group was lower than that in the control group, and the difference was statistically significant.

Subgroup analysis of the effect of fucoidan intervention on the number of tumors showed that the heterogeneity of different drug delivery methods and cancer types in the study group was less than $50 \%$, and these 2 variables affected the heterogeneity between studies. Different dosages, administration modes, and cancer types in the intervention group were lower than those in the control group, and the difference was statistically significant.

\section{Results of bias analysis}

An inverted funnel diagram was used to judge the publication bias of the included studies. Since the number of studies with tumor quantitative indicators was fewer than 10 , it was deemed too few a number, and the test efficiency too low to conduct publication bias evaluation. Only publication bias for tumor weight and tumor volume was 


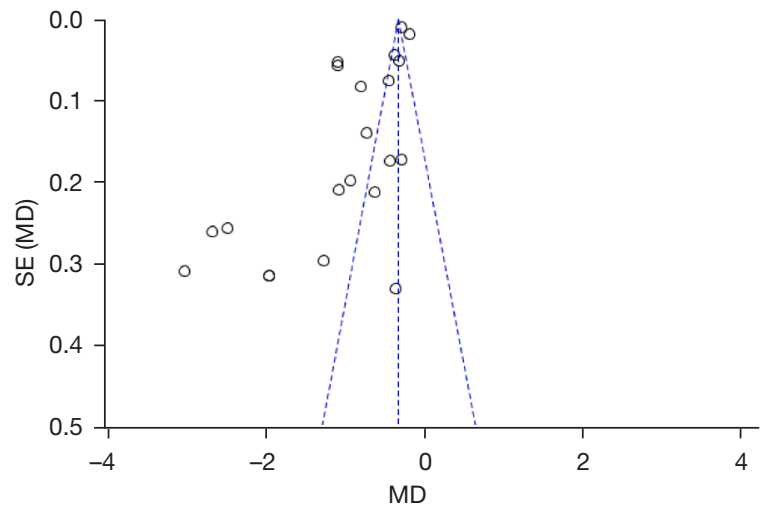

Figure 6 Funnel diagram of studies of tumor weight inhibition by fucoidan. SE, standard error; MD, mean difference.

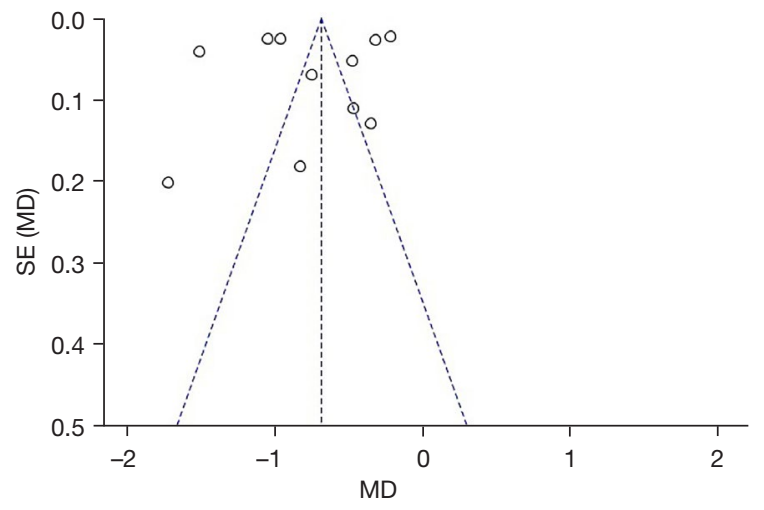

Figure 7 Funnel diagram of studies of tumor volume inhibition by fucoidan. SE, standard error; MD, mean difference.

evaluated (see Figures 6, 7).

As shown in Figure 6, the funnel chart of the literature included in the tumor weight analysis is asymmetric and skewed, and most of the selected studies are outside the funnel chart's $95 \%$ CI, indicating that those studies contained publication bias.

As displayed in Figure 7, the funnel chart structure of the literature included in tumor volume analysis is basically symmetrical; however, most of the selected studies were outside the funnel chart's $95 \% \mathrm{CI}$, indicating that those studies contained publication bias.

\section{Discussion}

This study involved a meta-analysis of animal studies exploring the effect of fucoidan on antitumor function in vivo in successfully established animal disease models. Based on the systematic evaluation of results, fucoidan can significantly inhibit tumor weight, tumor volume, and tumor number. In terms of the effect on tumor weight, the best effect was achieved with low dosage and intragastric administration in breast cancer models. In controlling tumor volume, the best effect was observed with low dosage and intraperitoneal injection in multiple myeloma models. In controlling the number of tumors, the best effect was achieved in melanoma models with high dosage and intraperitoneal injection. Different effects were shown across different tumor models, dosage, and administration methods, and the mechanism requires further study.

Meta-analysis showed that there was considerable heterogeneity among the studies. Subgroup analysis revealed that different dosages, modes of administration, and cancer types may have been the main reasons for heterogeneity between studies. As meta-analysis is based on data from the existing literature, conclusion reliability and literature bias are greatly influenced by the quality of the included studies (36). In this paper, although keywords and $\mathrm{MeSH}$ were used to search Chinese and international databases, the sources, components, concentrations, doses, administration methods, and intervention time among the 23 studies included in our analysis were inconsistent. Additionally, experimental animal subjects and models differed, and there was variation in index measurement methods and numerical units, leading to the heterogeneity and bias of the metaanalysis in this paper. Furthermore, the results included in the literature were all positive results, and there was no published literature with negative results. The results of bias analysis in this paper showed that all the included studies contained some publication bias. However, the results of sensitivity analysis showed good stability, which may reflect, to a certain extent, the reliability of the results showing the antitumor activity of fucoidan. In order to improve the repeatability of the research results, it is necessary to unify the dosage, components, and administration methods of fucoidan in future animal experiment research.

In this study, the SYRCLE animal experiment risk assessment tool was used to evaluate the quality of the included literature. In animal experiments, the randomization of animals has an important impact on the experimental results. If the animals are not randomly allocated, researchers may predict the behavior of each group of animals, resulting in implementation bias (36). In this study, only 11 studies $(8-12,14,15,17,18,26,30)$ utilized the randomized placement of animals. In addition, 
if the results were measured without randomization and the samples were only measured and evaluated in a certain period of time, there may be a risk of measurement bias (36). The literature included in this study did not report whether the outcome indicators were randomly evaluated. In animal experiments, the application of blind methods in the intervention, implementation, and result measurement stages differs from their execution in RCTs. Although there is no need to blind animals, most researchers involved in the process of intervention implementation are also animal breeders. If animal breeders are not blinded, subjective bias may arise in their expected experimental results (36). None of the studies included in this review described whether the breeders and researchers were blinded. More attention should be given to the implementation and full reporting of measures, including the balance of baseline characteristics, random result measurement, randomized animal placement, and blinding methods. This is especially important for promoting the establishment and implementation of animal experiment registries to enhance the transparency of the entire animal research process, promote the improvement of its quality, and ultimately improve the usefulness of basic research and provide a reliable theoretical basis for the transformation of basic research to clinical research.

In summary, this study investigated the effectiveness of the antitumor activity of fucoidan based on published animal experimental studies. The existing evidence showed that fucoidan inhibited the growth and spontaneous metastasis of tumors in numerous animal models, the effect of intraperitoneal injection at a low dose was best for most tumors, and the mechanism of fucoidan's antitumor activity warrants further research. However, as the design quality of the included studies was not high, heterogeneity and bias may have affected the accuracy of the results.

\section{Acknowledgments}

The authors gratefully acknowledge Ling-Ye Wang (School of Public Health, Xiamen University) and Dr. Hui-Qin Huang (Chinese Academy of Tropical Agricultural Sciences) for their excellent assistance in data processing. We would like to thank A. Muijlwijk and J. Jones for their help in polishing our paper.

Funding: This work was supported by the Key Research Project of Higher Education Institution of Hainan Province (Hnky2019ZD-23), the Natural Science Fundation of Hainan Province (821RC745).

\section{Footnote}

Reporting Checklist: The authors have completed the PRISMA reporting checklist. Available at https://dx.doi. org/10.21037/tcr-21-1733

Conflicts of Interest: All authors have completed the ICMJE uniform disclosure form (available at https://dx.doi. org/10.21037/tcr-21-1733). The authors have no conflicts of interest to declare.

Ethical Statement: The authors are accountable for all aspects of the work in ensuring that questions related to the accuracy or integrity of any part of the work are appropriately investigated and resolved.

Open Access Statement: This is an Open Access article distributed in accordance with the Creative Commons Attribution-NonCommercial-NoDerivs 4.0 International License (CC BY-NC-ND 4.0), which permits the noncommercial replication and distribution of the article with the strict proviso that no changes or edits are made and the original work is properly cited (including links to both the formal publication through the relevant DOI and the license). See: https://creativecommons.org/licenses/by-nc-nd/4.0/.

\section{References}

1. Dagenais GR, Leong DP, Rangarajan S, et al. Variations in common diseases, hospital admissions, and deaths in middle-aged adults in 21 countries from five continents (PURE): a prospective cohort study. Lancet 2020;395:785-94.

2. McCarthy CM. Discussion: The Impact of Radiation Therapy, Lymph Node Dissection, and Hormonal Therapy on Outcomes of Tissue Expander-Implant Exchange in Prosthetic Breast Reconstruction. Plast Reconstr Surg 2016;137:10-1.

3. Koh HK, Shin KH, Kim K, et al. Effect of Time Interval between Breast-Conserving Surgery and Radiation Therapy on Outcomes of Node-Positive Breast Cancer Patients Treated with Adjuvant Doxorubicin/ Cyclophosphamide Followed by Taxane. Cancer Res Treat 2016;48:483-90.

4. Jin JO, Zhang W, Du JY, et al. Fucoidan can function as an adjuvant in vivo to enhance dendritic cell maturation and function and promote antigen-specific $\mathrm{T}$ cell immune 
responses. PLoS One 2014;9:e99396.

5. Ai Zhengwen, Gui Min, Yu Peng, et al. Research Progress on the Effect of Fucoidin Structure on Its Function. Science and Technology of Food Industry 2019; 40:346-50+56

6. Ma Yujing, Yang Ling, He Rongxiang, et al. Physiological Functions of Fucoidan and Its Application in Animal Production. Chinese Animal Husbandry and Veterinary Medicine 2020;47:2404-12.

7. Hsu HY, Hwang PA. Clinical applications of fucoidan in translational medicine for adjuvant cancer therapy. Clin Transl Med 2019;8:15.

8. Yun CW, Yun S, Lee JH, et al. Silencing Prion Protein in HT29 Human Colorectal Cancer Cells Enhances Anticancer Response to Fucoidan. Anticancer Res 2016;36:4449-58.

9. Xue M, Ge Y, Zhang J, et al. Anticancer properties and mechanisms of fucoidan on mouse breast cancer in vitro and in vivo. PLoS One 2012;7:e43483.

10. Rui X, Pan HF, Shao SL, et al. Anti-tumor and antiangiogenic effects of Fucoidan on prostate cancer: possible JAK-STAT3 pathway. BMC Complement Altern Med 2017; 17:378

11. Liu S, Yang J, Peng X, et al. The Natural Product Fucoidan Inhibits Proliferation and Induces Apoptosis of Human Ovarian Cancer Cells: Focus on the PI3K/Akt Signaling Pathway. Cancer Manag Res 2020;12:6195-207.

12. Xue M, Liang H, Ji X, et al. Effects of fucoidan on gut flora and tumor prevention in 1,2-dimethylhydrazineinduced colorectal carcinogenesis. J Nutr Biochem 2020;82:108396.

13. Chen H, Cong Q, Du Z, et al. Sulfated fucoidan FP08S2 inhibits lung cancer cell growth in vivo by disrupting angiogenesis via targeting VEGFR2/VEGF and blocking VEGFR2/Erk/VEGF signaling. Cancer Lett 2016;382:44-52.

14. Xue M, Ji X, Xue C, et al. Caspase-dependent and caspaseindependent induction of apoptosis in breast cancer by fucoidan via the PI3K/AKT/GSK3 $\beta$ pathway in vivo and in vitro. Biomed Pharmacother 2017;94:898-908.

15. Xue M, Ge Y, Zhang J, et al. Fucoidan inhibited 4T1 mouse breast cancer cell growth in vivo and in vitro via downregulation of $\mathrm{Wnt} / \beta$-catenin signaling. Nutr Cancer 2013;65:460-8.

16. Han YS, Lee JH, Lee SH. Antitumor Effects of Fucoidan on Human Colon Cancer Cells via Activation of Akt Signaling. Biomol Ther (Seoul) 2015;23:225-32.
17. Duan Y, Li J, Jing X, et al. Fucoidan Induces Apoptosis and Inhibits Proliferation of Hepatocellular Carcinoma via the p38 MAPK/ERK and PI3K/Akt Signal Pathways. Cancer Manag Res 2020;12:1713-23.

18. Choo GS, Lee HN, Shin SA, et al. Anticancer Effect of Fucoidan on DU-145 Prostate Cancer Cells through Inhibition of PI3K/Akt and MAPK Pathway Expression. Mar Drugs 2016;14:126.

19. Zhang X, Wang J, Yang Y, et al. Study on the inhibitory effect of sea cucumber fucoidan on tumor growth and metastasis in mice and its mechanism. Chin Pharmacol Bull 2011;27:1098-103.

20. Zhang X, Wang J, Xu L, et al. Comparative Anti-tumor Effects of Fucoidan from Two Sea Cucumber Species on Spontaneous Metastasis of Lewis Lung Carcinoma in Mouse. Food Science 2012;33:251-5.

21. Zhang X. Study improing effect of fucans isolated from sea cucumbers on the type 2 diabetes and tumor metastasis mice [dissertation]. Qingdao: Ocean University of China, 2012:80.

22. Zhang X. Studies on purified fraction of fucoidan extracted from Ecklonia cava and its antitumor activity [dissertation]. Dalian: Dalian Ocean University, 2018:67.

23. Zhang T, Xue M, Liu J, et al. Protective effect of fucoidan on intestinal barrier in rats with breast cancer. Acta Nutrimenta Sinica 2018;40:59-63.

24. Yang Y. Study on anti-tumor effect and the mechanism of sea cucumber Fucoidan [dissertation]. Qingdao: Ocean University of China, 2012:98.

25. Wang Jingfeng, Zhang Xun, Li Hui, et al. Inhibitory effects of fucoidan, a sulfated polysaccharides from Cucumaria frondosa on experimental metastasis in mice. Chin J Mar Drugs 2012;31:14-18.

26. Liu F. Effect of fucoidan on angiogenesis indecued by human multiple myeloma cells [dissertation]. ChongQing: ChongQing Medical University, 2016:49.

27. Li Q, Yang L, Wang P, et al. Anti-tumor and immunomodulatory effects of Fucoidan on Lewis lung cancer-bearing mice. Chin J Mar Drugs 2013;32:12-6.

28. Xue M. Antitumor Effects of Fucoidan and the Related Mechanism in Vitro and in Vivo [dissertation]. Qingdao: Qingdao University, 2012:88.

29. Wang C, Cong J, Xian H, et al. Purification of Sulfated Polvsaccharide from Laminaria Japonica and Studies of its Anti-tumor Mechanism. Pharm J Chin PLA 2010;26:283-6.

30. Li Z. Determination method of kelp fucan sulfate and 
its antioxidant and antitumor activities [dissertation]. Qingdao: Ocean University of China, Qingdao Ocean University, 2001:54.

31. Cong Yao. Studies on the Structure of fucoidan from Kjellmaniella crassifolia and Antitumor activity of the degradation products [dissertation]. Dalian: Dalian Ocean University, 2017:50.

32. Wang P, Liu Z, Liu X, et al. Anti-metastasis effect of fucoidan from Undaria pinnatifida sporophylls in mouse hepatocarcinoma Hca-F cells. PLoS One 2014;9:e106071.

33. Wang ZJ, Xu W, Liang JW, et al. Effect of fucoidan on B16 murine melanoma cell melanin formation and apoptosis. Afr J Tradit Complement Altern Med 2017;14:149-55.

Cite this article as: Cao LM, Sun ZX, Makale EC, Du GK, Long WF, Huang HR. Antitumor activity of fucoidan: a systematic review and meta-analysis. Transl Cancer Res 2021;10(12):5390-5405. doi: 10.21037/tcr-21-1733
34. Tsai HL, Tai CJ, Huang CW, et al. Efficacy of LowMolecular-Weight Fucoidan as a Supplemental Therapy in Metastatic Colorectal Cancer Patients: A Double-Blind Randomized Controlled Trial. Mar Drugs 2017;15:122.

35. Yan Hongxue, Sun Zhanyi, Zhao Lili, et al. Research Progress in the Anticancer Activity of Fucoidan. Food Research and Development 2018;39:197-203.

36. Hooijmans CR, Rovers MM, de Vries RB, et al. SYRCLE's risk of bias tool for animal studies. BMC Med Res Methodol 2014;14:43.

37. Higgins JPT, Thomas J, Chandler J, et al. Cochrane Handbook for Systematic Reviews of Interventions version 6.2 (updated February 2021). Cochrane, 2021. Available online: https://training.cochrane.org/handbook 